\title{
Alfvén ship waves: high-m ULF pulsations in the magnetosphere generated by a moving plasma inhomogeneity
}

\author{
P. N. Mager and D. Yu. Klimushkin \\ Institute of Solar-Terrestrial Physics (ISTP), Russian Academy of Science, Siberian Branch, Irkutsk, P.O. Box 291, 664033, \\ Russia
}

Received: 8 October 2007 - Revised: 15 February 2008 - Accepted: 18 April 2008 - Published: 11 June 2008

\begin{abstract}
The generation of a high- $m$ Alfvén wave by substorm injected energetic particles in the magnetosphere is studied. The wave is supposed to be emitted by an alternating current created by the drifting particle cloud or ring current inhomogeneity. It is shown that the wave appears in some azimuthal location simultaneously with the particle cloud arrival at the same spot. The value of the azimuthal wave number is determined as $m \sim \omega / \omega_{d}$, where $\omega$ is the eigenfrequency of the standing Alfvén wave and $\omega_{d}$ is the particle drift frequency. The wave propagates westward, in the direction of the proton drift. Under the reasonable assumption about the density of the energetic particles, the amplitude of the generated wave is close to the observed amplitudes of poloidal ULF pulsations.
\end{abstract}

Keywords. Magnetospheric physics (MHD waves and instabilities) - Space plasma physics (Kinetic and MHD theory)

\section{Introduction}

Alfvén waves in the range of Pc3-5 can be categorized into the waves with large and small azimuthal wave numbers $m$. Recently, the high- $m$ waves were observed with CLUSTER (Eriksson et al., 2005, 2006; Schäfer et al., 2007) and radars (Fenrich et al., 1995; Yeoman et al., 2000; Wright et al., 2001; Baddeley et al., 2002). The low- $m$ waves are generated by the resonant interaction with the fast mode, propagating from the outer boundary of the magnetosphere. This generation mechanism is ineffective for pulsations with $m \gg 1$ (azimuthally small scale waves), because in this case only an exponentially small part of the fast mode energy penetrates into the magnetosphere (Glassmeier, 1995). Thus, other sources of the wave energy must be found. Substorm

Correspondence to: P. N. Mager

(p.mager@iszf.irk.ru) injected protons, drifting in the magnetosphere, appear to be good candidates. It is usually assumed that high- $m$ waves are excited by unstable proton populations with energies from 10 to $150 \mathrm{keV}$ by means of the drift-bounce instability (Karpman et al., 1977). An example of this unstable distribution function is the bump-on-tail distribution.

There are a number of arguments to support this suggestion. One of them is the coincidence of the directions of the azimuthal phase velocity of the high- $m$ pulsations and the proton drift velocity (Fenrich et al., 1995; Yeoman et al., 2000; Baddeley et al., 2005b; Glassmeier, 1980). Besides, both velocities depend on the radial coordinate almost in the same way (Allan et al., 1982, 1983). There is some evidence of statistical relations between the high- $m$ pulsations and ring current intensifications (Anderson, 1993; Yeoman et al., 2000). Association of the high- $m$ waves with nonmonotonic particle distributions have been observed by Hughes et al. (1978), Glassmeier et al. (1999), Wright et al. (2001) and statistically studied by Baddeley et al. (2002, 2004, 2005a,b).

The bump-on-tail distribution is usually supposed to be a result of a substorm injection: faster protons reach a given point on the azimuthal coordinate earlier than lower energy ones, so high-energy particles are added to the local background plasma at a higher rate than low-energy particles (Karpman et al., 1977; Glassmeier et al., 1999). Indeed, several cases were observed when the wave appeared in some azimuthal location simultaneously with the cloud of the particle injected during substorm arrival in the same spot (Chisham et al., 1992; Wright et al., 2001).

This mechanism of formation of the unstable distribution presupposes that the bump-on-tail is the natural consequence of the nonstationarity of the injection process. But the azimuthally drifting injected cloud (which can also be considered as a moving inhomogeneity of the ring current) represents an alternating current, which is able to emit the Alfvén wave itself (Akhiezer et al., 1967, 1975). In a magnetospheric context, this generation mechanism was first

Published by Copernicus Publications on behalf of the European Geosciences Union. 
considered by Zolotukhina (1974) and Guglielmi and Zolotukhina (1980). A similar problem has been considered for the magnetosphere of Jupiter, where its moon Io acts as the moving wave emitter (Neubauer, 1980). This mechanism resembles the generation of waves on the water surface by a moving ship (the analogy of whistlers and a ship wave has been considered by Gurnett, 1995).

Then, the drift-bounce instability does not explain some essential features of the high- $m$ waves. Thus, observed waves have definite $m$ numbers, although the weekly growth rate depends on this value (Mager and Klimushkin, 2005). Consequently, this instability cannot select a narrow range of the $m$ numbers, contrary to observations. Even the direction of the azimuthal phase velocity of the observed waves cannot be explained: the instability can generate the waves propagating in both azimuthal directions (Mager and Klimushkin, 2005). Finally, owing to transformation of poloidal Alfvén waves into toroidal ones, the instability will be favorable for amplification of toroidal rather than poloidal oscillations (Klimushkin, 2000, 2007; Klimushkin and Mager, 2004). Additional hints that some of the observed high- $m$ pulsations have been generated by nonstationary current are also reported by Pilipenko et al. (2001).

Our paper studies this generation mechanism in the Earth's magnetosphere. In contrast to the previous efforts in this direction (Akhiezer et al., 1967, 1975; Zolotukhina, 1974; Guglielmi and Zolotukhina, 1980; Neubauer, 1980; Pilipenko et al., 2001), where only a uniform background was considered, we explore a two-dimensionally inhomogeneous model of the magnetosphere with plasma and magnetic field non-uniformity along and across with field lines taken into account. Besides, as opposed to some earlier papers (Zolotukhina, 1974; Neubauer, 1980), the field lines are considered to intersect the highly conductive ionospheric plasma, which results in the emergence of a standing wave structure along the field lines. In contrast to our previous paper (Mager and Klimushkin, 2007), here we incorporated the dependence of the drift velocity on the radial coordinate: this velocity is supposed to grow with the L-shell.

\section{Formulation of the problem}

The method for setting up a problem is as follows. At some initial time instant $t=0$ a cloud of particles is injected into the magnetosphere. The particles are drifting in the azimuthal direction. It is required to obtain an expression for the wave amplitude and to find the spatio-temporal structure and polarization of the wave field.

The magnetosphere is considered as axially-symmetric and bounded by the highly conductive ionosphere of the Southern and Northern Hemispheres. The cloud of the injected particles is assumed to be narrowly localized in azimuth but distributed over the entire range of L-shells, i.e. in the radial direction. The source is also distributed along field lines between conjugated points of the ionosphere. Zolotukhina (1974) and Neubauer (1980) considered the source compact (localized) in all directions.

The drift angular velocity $\omega_{d}$ is assumed to be much less than the characteristic Alfvén eigenfrequency. In particular, it means that the velocity of the source is much less than the Alfvén speed. It corresponds to the energies of the order of several tens of $\mathrm{keV}$.

Besides, we assumed the drift angular velocity $\omega_{d}$ to increase with the radial coordinate (L-shell). For simplicity, we even put $\omega_{d}$ to be proportional to the radial coordinate, but we suppose that our results are generally valid for any increasing functions $\omega_{d}(L)$. The most common instance is probably the case when the angular drift speed decreases with the L-shell, because in this case the injected protons conserve the first adiabatic invariant. But, as we will show in the last section, the case considered in the present paper happens to be more interesting, since it shows a rather unexpected feature of the wave field temporal evolution, which can be used for the verification of our model. On the other hand, this case can also be realized in the magnetosphere under some conditions (e.g. Southwood, 1980), when there is loss of injected protons. The case when injected protons have a drift velocity constant with radius has been considered in our earlier paper (Mager and Klimushkin, 2007).

Our approach is based on the theory of eigenoscillations of the axisymmetric magnetosphere developed by Leonovich and Mazur (1997) and Klimushkin et al. (2004) and uses the general approach by Akhiezer et al. (1967) and Akhiezer et al. (1975). A time-dependent external current (formed by the moving charged particle cloud) is assumed to be the source term of the wave equation. The particle density of the cloud is considered to be small compared to the background density, which allows us to consider the waves in the linear approximation. The current (wave generator) is considered to be given, that is we neglect the feedback of the generated wave on the current. This assumption is probably valid at the earlier stages of the wave field evolution (Akhiezer et al., 1967). On the other hand, we do not consider the evolution within the first few bounce periods, when the cloud is continuing to spread along the field lines and the parallel structure of the wave has not been settled yet. This time is very short because the drift velocity is much less than the thermal particle velocity, so this limitation is not too severe.

\section{Main equations}

Let us introduce a curvilinear coordinate system $\left\{x^{1}, x^{2}, x^{3}\right\}$, in which the field lines play the role of the coordinate lines $x^{3}$, i.e. such lines, along which the other two coordinates are invariable (recall that the superscripts and subscripts denote contravariant and covariant coordinates, respectively). In this coordinate system the stream lines are the coordinate lines $x^{2}$, and the surfaces of constant pressure (magnetic shells) 
are the coordinate surfaces $x^{1}=$ const (Fig. 1). The coordinates $x^{1}$ and $x^{2}$ have the role of the radial and azimuthal coordinates, and to represent them we shall use the McIlwain parameter $L$ and the azimuthal angle $\varphi$, respectively. It is convenient to choose a direction of the azimuthal coordinate coinciding with the proton drift direction. In order for the coordinate system to remain right-handed, the $x^{3}$ axis must be directed opposite to the ambient magnetic field. The physical length along a field line is expressed in terms of an increase of the corresponding coordinate as $d l_{3}=\sqrt{g_{3}} d x^{3}$, where $g_{3}$ is the component of the metric tensor, and $\sqrt{g_{3}}$ is the Lamé coefficient. Similarly, $d l_{1}=\sqrt{g_{1}} d x^{1}$, and $d l_{2}=\sqrt{g_{2}} d x^{2}$. The determinant of the metric tensor is $g=g_{1} g_{2} g_{3}$. The equilibrium values of the magnetic field and plasma density are designated as $B$ and $\rho ; \xi$ is the displacement of plasma from the equilibrium position, $E, b$ and $j$ are the electric field, magnetic field, and current of the wave. The source of the oscillations is a nonstationary external (azimuthal) current $\boldsymbol{j}_{\text {ext }}$, formed by drifting substorm injected particles. The stationary current is absent in the cold plasma approximation.

In this approximation, the linearized equation of small oscillations takes the form

$\rho \frac{\partial^{2} \boldsymbol{\xi}}{\partial t^{2}}-\frac{1}{c} \boldsymbol{j} \times \boldsymbol{B}=0$.

The electrodynamic values are interconnected by the equations

$\nabla \times \boldsymbol{b}=\frac{4 \pi}{c} \boldsymbol{j}+\frac{4 \pi}{c} \boldsymbol{j}_{\mathrm{ext}}$

(Ampère law),

$\nabla \times \boldsymbol{E}=-\frac{1}{c} \frac{\partial \boldsymbol{b}}{\partial t}$

(Maxwell equation),

$\boldsymbol{E}=\frac{1}{c} \frac{\partial \boldsymbol{\xi}}{\partial t} \times \boldsymbol{B}$

(frozen-in condition). It is worth noting that the outer current appeared only in Eq. (2) (Akhiezer et al., 1975). Using Eqs. (1-4), we obtain the equation for the wave electric field $E$ :

$\frac{1}{A^{2}} \frac{\partial^{2} \boldsymbol{E}}{\partial t^{2}}-\nabla \times \nabla \times \boldsymbol{E}=-\frac{4 \pi}{c^{2}} \frac{\partial \boldsymbol{j}_{\mathrm{ext}}}{\partial t}$,

where $A=B / \sqrt{4 \pi \rho}$ is the Alfvén speed. Due to infinite plasma conductivity, the parallel electric field is absent, thus the wave's electric field lies on surfaces orthogonal to field lines. The electric field of the Alfvén mode can be represented in the form

$\boldsymbol{E}=-\nabla_{\perp} \Phi$,

where $\Phi$ is a scalar function ("potential"), and $\nabla_{\perp}$ is the transverse nabla operator. Let us substitute Eq. (6) into

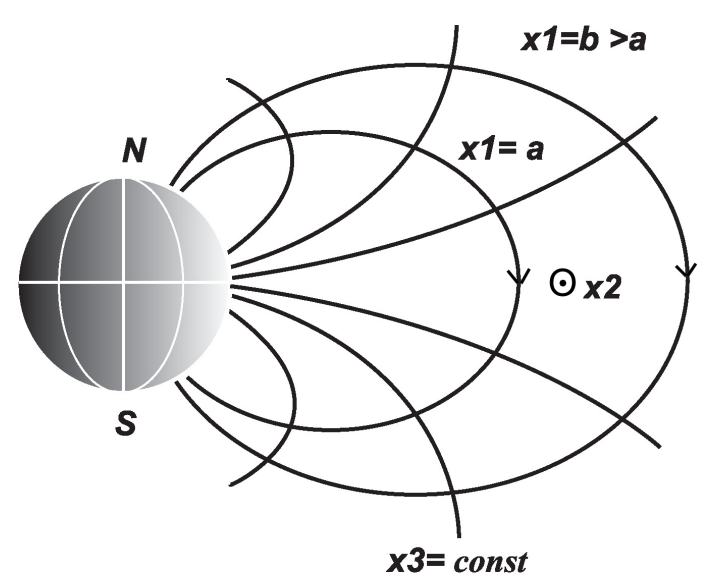

Fig. 1. The coordinate system.

Eq. (5) and act on the obtained expression by the operator $\nabla_{\perp}$. As a result, we obtain the equation

$\mathcal{L}_{A} \Phi=-\frac{4 \pi}{c^{2}} \sqrt{g} \frac{\partial}{\partial x^{2}} \frac{\partial}{\partial t} j_{\text {ext }}^{2}$.

Here $j_{\text {ext }}^{2}=j_{\text {ext }} / \sqrt{g_{2}}$ is the contra-variant azimuthal projection of the vector $\boldsymbol{j}_{\mathrm{ext}}$, and

$$
\begin{aligned}
\mathcal{L}_{A} & =\frac{\partial}{\partial x^{1}}\left[-\frac{\sqrt{g}}{g_{1}} \frac{1}{A^{2}} \frac{\partial^{2}}{\partial t^{2}}+\frac{\partial}{\partial x^{3}} \frac{g_{2}}{\sqrt{g}} \frac{\partial}{\partial x^{3}}\right] \frac{\partial}{\partial x^{1}} \\
& +\frac{\partial}{\partial x^{2}}\left[-\frac{\sqrt{g}}{g_{2}} \frac{1}{A^{2}} \frac{\partial^{2}}{\partial t^{2}}+\frac{\partial}{\partial x^{3}} \frac{g_{1}}{\sqrt{g}} \frac{\partial}{\partial x^{3}}\right] \frac{\partial}{\partial x^{2}}
\end{aligned}
$$

is an Alfvén differential operator. Thus, we have an inhomogeneous differential equation which describes Alfvén oscillations generated by the external current. The boundary conditions are chosen as

$\left.\Phi\right|_{x^{1}, x^{2} \rightarrow \pm \infty}=0,\left.\quad \Phi\right|_{x_{ \pm}^{3}}=0$.

Here the second condition corresponds to the full wave reflection from the ionosphere $\left(x_{ \pm}^{3}\right.$ denotes the points of the intersection of the field line with the ionosphere).

The cloud of drifting particles comprising the external current is assumed to be narrowly localized in azimuth, that is the contra-variant azimuthal projection of the external current

$j_{\mathrm{ext}}^{2}=e n_{0} \omega_{d} \delta\left(\varphi-\omega_{d} t\right) \Theta(t)$,

where $\omega_{d}\left(x^{1}\right)$ is the bounce-averaged angular drift velocity, $e$ and $n_{0}$ are the electric charge and number density of the particles, $\Theta(t)$ is the Heaviside theta-function, denoting the instant when the source is "switched on" (injection of particles into the magnetosphere), $\varphi$ is the azimuthal angle, which can be used as the $x^{2}$ coordinate. The physical component of the current can be obtained by using the linear drift velocity 


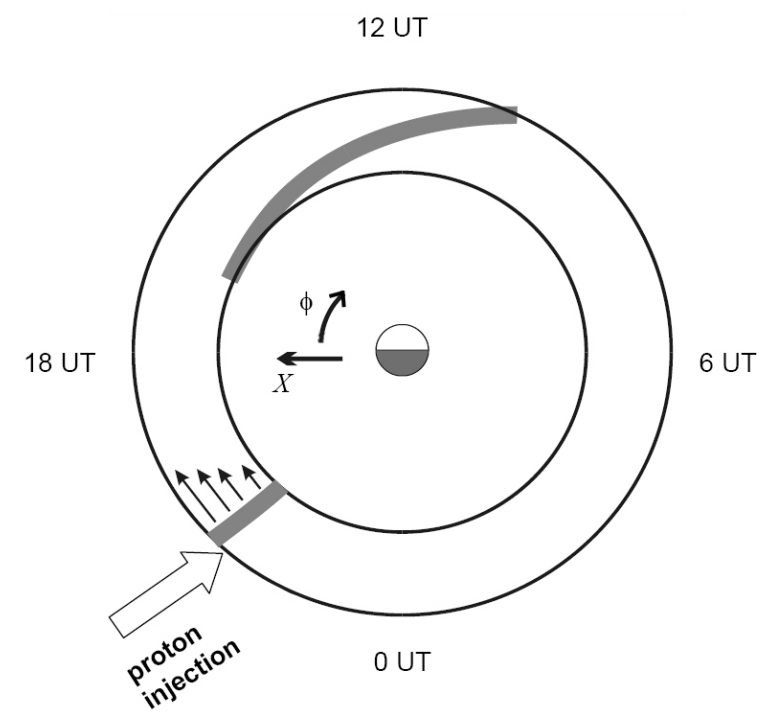

Fig. 2. The model of the source.

$V=\sqrt{g_{2}} \omega_{d}$ instead of the angular velocity in Eq. (9). The injection takes place at the instant $t=0$. For simplicity, we put the drift velocity to be proportional to the radial coordinate, $\omega_{d}\left(x^{1}\right)=\Omega_{d} x^{1}$, where $\Omega_{d}$ does not depend on $x^{1}$. As is easy to see that in the course of time the cloud will be stretched into a spiral (Fig. 2).

In order to solve the wave Eq. (7), we perform the Fouriertransform of this equation over $\varphi$ and $t$ (see Appendix A). As a result, we obtain a differential equation only with respect to two variables, $x^{1}$ and $x^{3}$ :

$\hat{L}_{A} \Phi_{m \omega}=\tilde{q}_{m \omega}$,

where $\omega$ and $m$ are the parameters of the Fourier transform over time (frequency) and azimuthal angle (azimuthal wave number), and

$$
\begin{aligned}
\tilde{q}_{m \omega} & =-2 m \omega \sqrt{g} \frac{e n_{0} \omega_{d}}{c^{2}} \\
& \times \frac{1}{2 \pi} \int_{-\infty}^{+\infty} \Theta\left(t^{\prime}\right) \exp \left(i \omega t^{\prime}-i m \omega_{d} t^{\prime}\right) d t^{\prime} .
\end{aligned}
$$

In Eq. (10), $\hat{L}_{A}$ is the Fourier-image of the Alfvénic operator $\mathcal{L}_{A}$ analogous to the Alfvénic operator for the monochromatic wave with frequency $\omega$ and azimuthal wave number $m$, defined as

$$
\hat{L}_{A} \equiv \frac{\partial}{\partial x^{1}} \hat{L}_{T}(\omega) \frac{\partial}{\partial x^{1}}-m^{2} \hat{L}_{P}(\omega),
$$

where

$$
\hat{L}_{T}(\omega)=\frac{\partial}{\partial x^{3}} \frac{g_{2}}{\sqrt{g}} \frac{\partial}{\partial x^{3}}+\frac{\sqrt{g}}{g_{1}} \frac{\omega^{2}}{A^{2}}
$$

is the toroidal mode operator, and

$$
\hat{L}_{P}(\omega)=\frac{\partial}{\partial x^{3}} \frac{g_{1}}{\sqrt{g}} \frac{\partial}{\partial x^{3}}+\frac{\sqrt{g}}{g_{2}} \frac{\omega^{2}}{A^{2}},
$$

is the poloidal mode operator. The eigenvalues of these operators with the boundary condition on the ionosphere (8) are denoted $\Omega_{T N}$ and $\Omega_{P N}$, respectively. They are called the toroidal and poloidal eigenfrequencies since they characterize the purely azimuthal (toroidal) and radial (poloidal) oscillations of field lines (e.g. Klimushkin et al., 2004; Leonovich and Mazur, 1997).

\section{The structure of a single Fourier harmonic}

The method of the solution of Eq. (10) has been developed by Klimushkin et al. (2004). As was shown there, the function $\Phi_{m \omega}$ can be represented as

$\Phi_{m \omega} \approx R_{N}\left(x^{1}\right) T_{N}\left(x^{1}, x^{3}\right)$,

where $T_{N}\left(x^{1}, x^{3}\right)$ is an eigenfunction of the toroidal operator $\hat{L}_{T}$, defining a longitudinal structure of the $N$-th harmonic standing between ionospheres. The normalization condition is

$$
\left\langle\frac{\sqrt{g}}{g_{1}} \frac{T_{N}^{2}}{A^{2}}\right\rangle=1
$$

(here the angle brackets designate integration along the field line between the ionospheres, $\left.\langle\ldots\rangle=\int_{x_{-}^{3}}^{x_{+}^{3}}(\ldots) d x^{3}\right)$. The function $R_{N}\left(x^{1}\right)$ describes the structure of this harmonic across the magnetic shells.

Let us introduce some definitions. The toroidal and poloidal eigenfrequencies are functions of the radial coordinate $x^{1}$. If the wave frequency $\omega$ is fixed, we can introduce the notions of toroidal $x_{T N}^{1}$ and poloidal $x_{P N}^{1}$ magnetic shells determined as solutions of the equations

$\omega=\Omega_{T N}\left(x^{1}\right)$

and

$\omega=\Omega_{P N}\left(x^{1}\right)$,

respectively. The distance between these shells is designated as $\Delta_{N}=x_{T N}^{1}-x_{P N}^{1}$ (in a cold plasma, $\Delta_{N}>0$ ). In the major part of the magnetosphere, the functions $\Omega_{P N}\left(x^{1}\right)$ and $\Omega_{T N}\left(x^{1}\right)$ are monotonically decreasing with the characteristic scale $l$, which has the same order of magnitude as the size of the magnetosphere. For the sake of simplicity, we can then avail ourselves of the linear expansions

$\Omega_{T N}\left(x^{1}\right)=\Omega_{0}\left(1-\frac{x^{1}}{l}\right)$

and

$\Omega_{P N}\left(x^{1}\right)=\Omega_{0}\left(1-\frac{x^{1}+\Delta_{N}}{l}\right)$. 
After substitution of expressions (13) and (14) into Eqs. (15) and (16), we obtain

$x_{T N}^{1}(\omega)=l\left(1-\frac{\omega}{\Omega_{0}}\right)$,

$x_{P N}^{1}(\omega)=x_{T N}^{1}-\Delta_{N}$.

Further, if we substitute the function $\Phi_{m \omega}\left(x^{1}, x^{3}\right)$ from Eq. (11) into Eq. (10), we obtain the ordinary differential equation defining the radial structure of the wave field:

$$
\begin{aligned}
\frac{\partial}{\partial x^{1}}\left(x^{1}-x_{T N}^{1}(\omega)\right) \frac{\partial}{\partial x^{1}} R_{N} & -\frac{m^{2}}{L^{2}}\left(x^{1}-x_{P N}^{1}(\omega)\right) R_{N} \\
& =m q\left(x^{1}, \omega, m\right) .
\end{aligned}
$$

See Klimushkin et al. (2004), Leonovich and Mazur (1997) for more detail. Here

$q\left(x^{1}, \omega, m\right)=\frac{q_{0}}{2 \pi} \int_{-\infty}^{+\infty} \Theta\left(t^{\prime}\right) \exp \left(i \omega t^{\prime}-i m \Omega_{d} x^{1} t^{\prime}\right) d t^{\prime}$,

$q_{0}=-\frac{e l}{c^{2}} \frac{\omega_{d}}{\Omega_{0}}\left\langle n_{0} \sqrt{g} T_{N}\right\rangle$

The solution of the Eq. (17) satisfying the boundary condition (8) is

$$
\begin{aligned}
& R_{N}\left(x^{1}, \omega, m\right) \\
& =i q_{0} L \int_{-\infty}^{+\infty} d \kappa \int_{-\infty}^{+\infty} d t^{\prime} \frac{\Theta\left(\kappa+m \Omega t^{\prime}\right) \Theta\left(t^{\prime}\right)}{\sqrt{\left(\kappa^{2}+m^{2} \frac{l^{2}}{L^{2}}\right)\left(\frac{L^{2}}{l^{2}} \Omega^{2} t^{\prime 2}+1\right)}} \\
& \times \exp \left[i \omega\left(t^{\prime}+\frac{m \Omega}{\Omega_{0}} t^{\prime}+\frac{\kappa}{\Omega_{0}}\right)-i m \Omega t^{\prime}+i \kappa(\xi-1)\right. \\
& \left.+i m \delta_{N} \frac{l}{L}\left(\arctan \frac{\kappa L}{m l}+\arctan \Omega t^{\prime} \frac{L}{l}\right)\right]
\end{aligned}
$$

(see Appendix B). The notations are:

$\xi=x^{1} / l, \quad \delta_{N}=\Delta_{N} / l, \quad \Omega=\Omega_{d} l$.

\section{The structure and evolution of the wave field}

With the solution (10), we can solve the wave equation (7) by means of the reverse Fourier transform:

$\Phi\left(x^{1}, x^{2}, x^{3}, t\right)=\int_{-\infty}^{+\infty} d \omega \int_{-\infty}^{+\infty} d m \Phi_{m \omega} e^{i m \varphi-i \omega t}$.

Thus, according to Eqs. $(20,11)$, the solution of the wave equation (7) is

$\Phi\left(x^{1}, x^{2}, x^{3}, t\right)=\mathcal{R}_{N}\left(x^{1}, x^{2}, t\right) T_{N}\left(x^{1}, x^{3}\right)$,

where the function

$\mathcal{R}_{N}\left(x^{1}, x^{2}, t\right)=\int_{-\infty}^{+\infty} d \omega \int_{-\infty}^{+\infty} d m R_{N}(m, \omega) e^{i m \varphi-i \omega t}$ defines both the transverse structure of the wave and its evolution. The expression for $\mathcal{R}_{N}$ can be reduced to the form (see Appendix C):

$$
\begin{aligned}
& \mathcal{R}_{N}\left(x^{1}, x^{2}, t\right)=i q_{0} L \mu \\
& \times \int_{-\infty}^{+\infty} d m \int_{-\infty}^{+\infty} d \kappa \Theta(\kappa+m \Omega t) \Theta\left(\Omega_{0} t-\kappa\right) e^{i \Psi(m, \kappa)} \\
& \times\left[\left(\kappa^{2}+\frac{m^{2} l^{2}}{L^{2}}\right)\left(\frac{L^{2}\left(\Omega_{0} t-\kappa\right)^{2}}{l^{2}}+(\mu+m)^{2}\right)\right]^{-1 / 2}
\end{aligned}
$$

where

$$
\begin{aligned}
& \Psi(m, \kappa)=m \varphi-m \frac{\Omega_{0} t-\kappa}{\mu+m}+\kappa(\xi-1) \\
& +m \delta_{N} \frac{l}{L}\left(\arctan \frac{\kappa L}{m l}+\arctan \frac{L}{l} \frac{\Omega_{0} t-\kappa}{\mu+m}\right) .
\end{aligned}
$$

Since the drift angular velocity $\omega_{d}$ is assumed to be much less than the Alfvénic eigenfrequencies $\Omega_{T N}$ and $\Omega_{P N}$, the large parameter $\mu=\Omega_{0} / \Omega$ appears. In this case the double integral (23) can be evaluated by means of the stationary phase method (see Appendix D). Having done this calculation, we finally obtain the approximate expression for $\mathcal{R}_{N}$ :

$\mathcal{R}_{N}=\mathcal{A}_{0} e^{i \Psi_{0}}$

where the wave phase is

$$
\begin{aligned}
\Psi_{0} & \equiv \Psi\left(\kappa_{0}, m_{0}\right)=m_{0}\left(x^{1}\right) \varphi-\Omega_{T N}\left(x^{1}\right) t \\
& +k_{a}\left(x^{1}\right) \Delta_{N}\left[\arctan \frac{k_{r}\left(x^{1}, \varphi, t\right)}{k_{a}\left(x^{1}\right)}+\arctan \frac{L \varphi}{x^{1}}\right],
\end{aligned}
$$

and the amplitude is

$$
\mathcal{A}_{0}=\frac{i 2 \pi q_{0} L \mu \Theta(\varphi) \Theta\left(\omega_{d}\left(x^{1}\right) t-\varphi\right)}{\left[k_{r}\left(x^{1}, \varphi, t\right)^{2}+k_{a}\left(x^{1}\right)^{2}\right]^{1 / 2}\left[x^{1^{2}}+L^{2} \varphi^{2}\right]^{1 / 2}} .
$$

The factor $\Theta\left(\omega_{d} t-\varphi\right)$ in Eq. (27) shows that the wave field is absent before the source in this approximation. Also, the following designations are introduced here: the radial component of the wave vector

$k_{r}\left(x^{1}, \varphi, t\right)=\frac{\mu}{l}\left(\Omega t-\frac{\varphi l^{2}}{x^{1^{2}}}\right)$,

the azimuthal component of the wave vector and the azimuthal wave number

$$
k_{a}\left(x^{1}\right)=\frac{\mu}{L} \frac{l-x^{1}}{x^{1}}=\frac{m_{0}\left(x^{1}\right)}{L}, m_{0}\left(x^{1}\right)=\frac{\Omega_{T N}\left(x^{1}\right)}{\omega_{d}\left(x^{1}\right)} .
$$



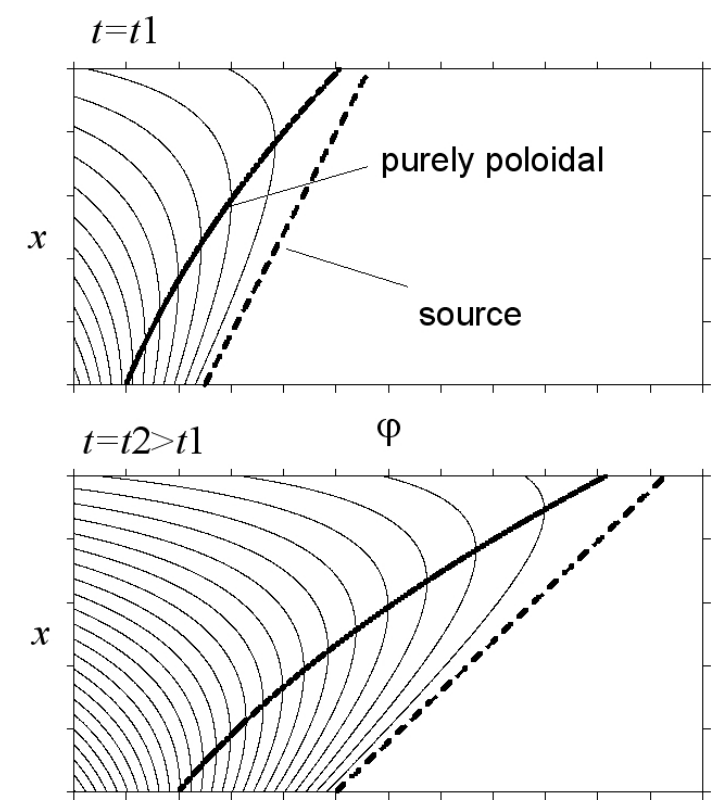

Fig. 3. The lines of the constant phase for different time instants.

\section{Discussion}

Let us discuss the main features of the solution obtained. The angular frequency of the wave is defined as

$\omega=\frac{\partial \Psi}{\partial t}=\Omega_{T N}-\frac{\Delta_{N}}{l} \Omega_{0}\left(1+\frac{k_{r}}{k_{a}}\right)^{-1} \approx \Omega_{T N}$.

Hence, the frequency depends on the radial coordinate $x^{1}$. Moreover, in the course of time $\omega$ is changed from $\Omega_{P N}$ to $\Omega_{T N}$. If we define the projection of the wave vector by means of a similar procedure, that is as partial derivatives of the phase (26) as $k_{r}=\partial \Psi / \partial r, k_{a}=\partial \Psi / \partial \varphi$, then their expression will differ from Eqs. $(28,29)$ only by small values, proportional to the parameter $\delta_{N}=\Delta_{N} / l \ll 1$. That means that Eqs. $(28,29)$ can be safely used for needs of the qualitative discussion. As is seen from those expressions, the azimuthal wave number $m$ also depends on time, changing from $m_{P}=\Omega_{P N} / \omega_{d}$ to $m_{T}=\Omega_{T N} / \omega_{d}$. But this dependence is rather weak due to a small difference between toroidal and poloidal eigenfrequencies in cold plasma.

Much more important is a strong time dependence of the wave vector radial component. Near the line $\Omega t-\varphi\left(l / x^{1}\right)^{2}=0$, this value is very small, $k_{r} \ll k_{a}$. As the source is moving away from the points on this curve, the radial component increases (Fig. 3). But according to Eqs. (6) and (25), the ratio between $k_{r}$ and $k_{a}$ defines the wave polarization: $\left|E_{a} / E_{r}\right|=k_{a} / k_{r}$. Thus, just after the generation the wave has a mixed polarization, and as the wave moves farther and farther away from the source, it becomes poloidallypolarized $\left(E_{r} \ll E_{a}\right)$. Further, the wave finally transforms

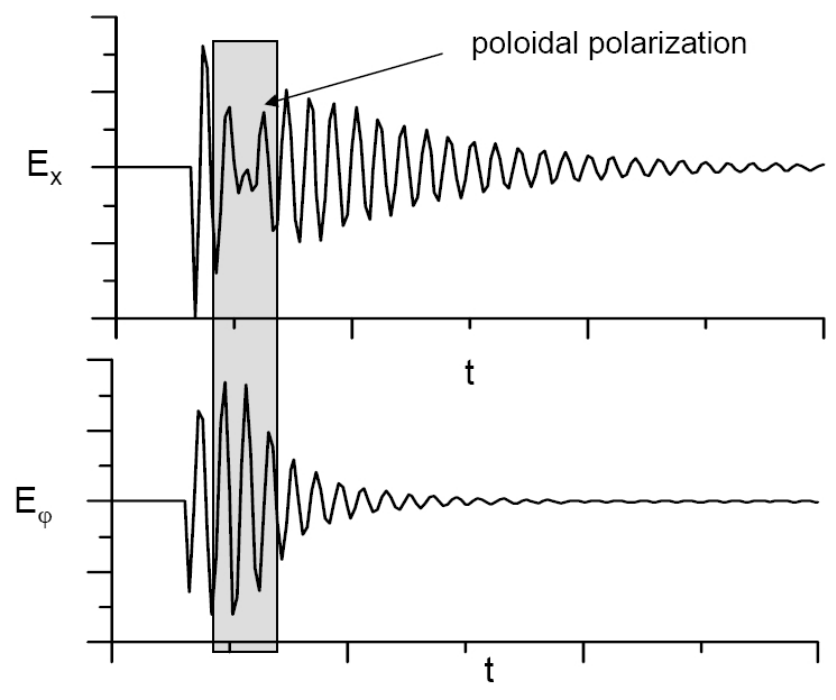

Fig. 4. The wave electric field radial and azimuthal components at fixed $x^{1}$ and $\varphi$ coordinates.

into a toroidally-polarized one $\left(E_{r} \gg E_{a}\right)$. The characteristic transformation time is

$\tau=2 \frac{m_{0} l}{\Omega_{0} L}$.

It is during this time span that the wave remained poloidallypolarized. An analogous transformation takes place also in the case of impulsive excitation (see, e.g. Klimushkin and Mager (2004)) with a similar transformation time $\tau \sim m / \omega$.

Such a double change of polarization was absent in the model where the drift velocity does not depend on the radial coordinate (Mager and Klimushkin, 2007). In the model considered in the present paper the particle drift velocity grows with distance from the Earth. Consequently, the source stretches into strips at an acute angle to the surface $x^{1}=$ const (as usual, the angle is measured counter-clockwise). Directly near the source the lines of the constant phase are parallel to the source (see Fig. 3). This means the presence of both radial azimuthal components of the wave vector, that is, mixed wave polarization. Further evolution is accompanied by turning of the lines of the constant phase counter-clockwise. At some time instant, there appears a point on a line of the constant phase where this line is tangent to the $x^{2}=$ const axis. At this point, the wave vector radial component equals zero, that is, the mode is poloidally polarized. Thus, the mode is poloidally polarized on the line passing through these points. Further, at a given point the constant phase line becomes inclined at an obtuse angle to the $x^{1}=$ const surface, that is, the wave vector radial component appears again. In the course of time, the angle is increasing, i.e. the ratio $\left|k_{r} / k_{a}\right|$ is increasing too and the wave becomes more and more toroidal. The above mentioned features are illustrated in Fig. 4, where the temporal evolution of the radial and azimuthal components 


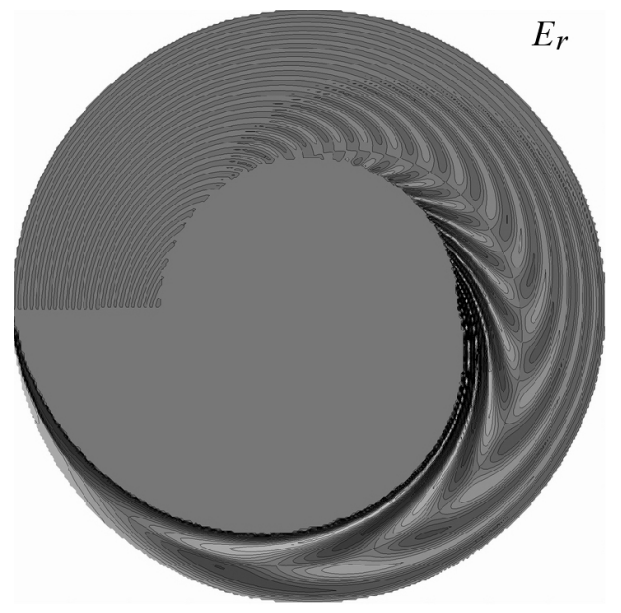

Fig. 5. The radial component of the electric field.

of electric field is shown, and also in Figs. 5-7, where the full wave structure is depicted. To compute these figures, wave attenuation due to finite ionospheric conductivity was taken into account.

With damping taken into account, Eq. (17) is written as

$$
\begin{aligned}
& \frac{\partial}{\partial x^{1}}\left(x^{1}-x_{T N}^{1}(\omega)+i \frac{l \gamma}{\Omega_{0}}\right) \frac{\partial}{\partial x^{1}} R_{N}- \\
& -\frac{m^{2}}{L^{2}}\left(x^{1}-x_{P N}^{1}(\omega)+i \frac{l \gamma}{\Omega_{0}}\right) R_{N}=m q(\omega, m),
\end{aligned}
$$

where $\gamma$ is a decrement of ionospheric damping. Having performed the manipulations described above, we obtain that in this case the expression (25) obtains one additional factor, namely

$\mathcal{R}_{N}=\mathcal{A}_{0} e^{-\frac{\gamma}{\omega_{d}}\left(\omega_{d} t-\varphi\right)} e^{i \Psi_{0}}$.

Based upon this expression, we evaluate the width of the localization region across magnetic shells. It is evident that the amplitude will be highest just near the source, i.e. in the vicinity of the curve $\omega_{d}\left(x^{1}\right) t-\varphi=0$. Thus, at $\gamma t>1$ the width of the localization region will be

$\Delta L=\frac{L}{\gamma t-1}$.

As is seen from this expression, the wave becomes more localized with time. For example, for the shell $L=6$, at $\gamma=0.1 \omega$ and $t=10 T$ (where $T$ is wave period), the width of the localization region is $\Delta L \approx 1 R_{E}$. As observations show, high- $m$ Alfvén waves often have a localization width of $\sim 1 R_{E}$.

Let us evaluate the wave amplitude. On the basis of the Eqs. $(18,21$, and 25), we obtain

$\Phi \sim \frac{2 \pi e L^{2}}{c^{2} \mu}\left\langle n_{0} \sqrt{g} T_{N}\right\rangle T_{N} \sim \frac{2 \pi e n_{0} L^{2} A^{2}}{c^{2} \mu}$.

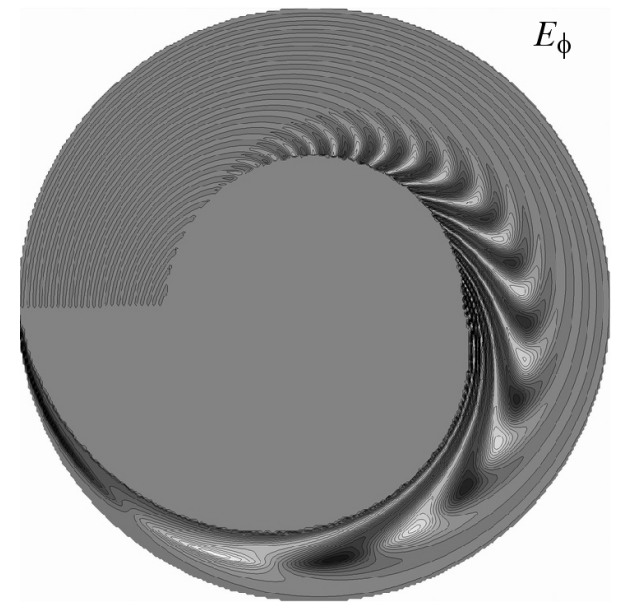

Fig. 6. The azimuthal component of the electric field.

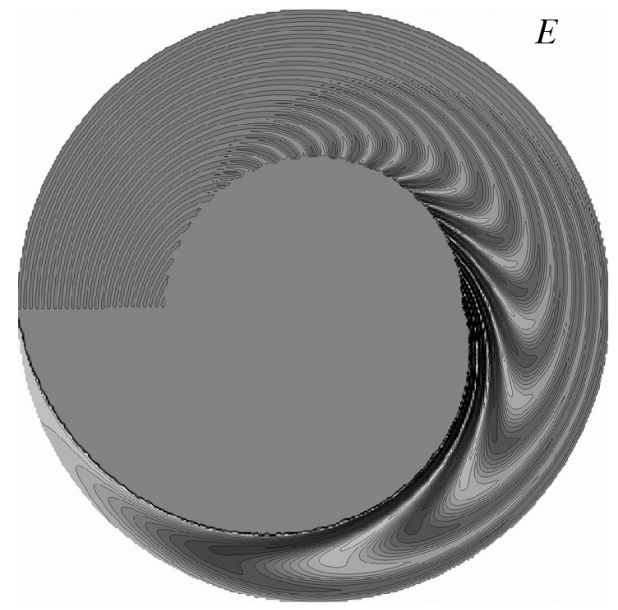

Fig. 7. The full electric field of the wave.

For this ordering, we used the normalization condition (12) for the eigenfunction $T_{N}$, which implies that $T_{N} \sim A / L$ and $\left\langle n_{0} \sqrt{g} T_{N}\right\rangle \sim n_{0} A L$. Further, from Eq. (6) we find the wave electric and magnetic fields:

$E \sim \frac{\mu}{L} \Phi \sim \frac{2 \pi e n_{0} L A^{2}}{c^{2}}$,

$b \sim \frac{c}{A} E \sim \frac{2 \pi e n_{0} L A}{c}$.

Now, using Eq. (34), we can find which proton number density $n_{0}$ in the drifting cloud is necessary for the generation of Alfvén waves with observed magnetic field amplitudes $b$ :

$n_{0} \sim \frac{b c}{2 \pi e L A}$.

The amplitude of azimuthally-small scale Alfvén waves in the magnetosphere can reach values of $b \sim 40 \times 10^{-5} \mathrm{Gs}$, their 
periods are of the order of $100 \mathrm{~s}$, azimuthal wave numbers $m \sim 20-100$, they are frequently observed in the vicinity of $L \sim 6$ magnetic shell (Chisham et al., 1992). The characteristic value of the Alfvén speed is $A \sim 1000 \mathrm{~km} / \mathrm{s}$. To generate Alfvén waves with such properties the number density must be $n_{0} \sim 10^{-2} \mathrm{sm}^{-3}$, which is much less than the background (cold) plasma density; the particle energy $\epsilon$ determining the velocity of proton drift in an inhomogeneous magnetic field (the source speed $\Omega$ ) must be about $50 \mathrm{keV}$ (the azimuthal wave number of the generated wave $m \sim \omega / \Omega$ ). The particles with such energies are often observed simultaneously with high- $m$ oscillations.

\section{Conclusions}

Let us outline the general picture of the Alfvén wave generation by the moving plasma inhomogeneity. At a given point in the azimuthal coordinate, the wave appears just after the source arrival. Under the realistic assumptions of the particle energy and density in the moving source, the amplitudes of the generated oscillations are close to those really observed. The direction of the wave propagation coincides with the direction of the source movement, the wave polarization is intermediate between poloidal and toroidal. As the source moves off the given point, the wave transforms first into a poloidal and then into toroidal wave. Note the oscillatory structure of the wave field behind the source, which is a consequence of the finite size of the cloud along the field line (as we assumed the source to be distributed between the conjugated points of the ionosphere). Earlier, Zolotukhina (1974) and Neubauer (1980) showed that the Alfvén wave excited by a compact moving cloud (that is, by a source much shorter than the field line) constituted current (Alfvén) wings, spreading from the source. Since a finite source can be represented as a superposition of many compact sources, the oscillatory structure obtained in this paper can be viewed as a result of the interference of such wings. Just after the injection of the cloud, when the cloud is continuing to spread along the field line (within few first bounce periods), the wave field probably resembles pure Alfvén wings. However, the evolution just after the injection has not been considered in this paper.

The mechanism considered in the paper provides a way for explaining the features of the observed azimuthally small scale waves mentioned in the Introduction:

1. The azimuthal wave number $m$ is fully determined by the eigenfrequency of the wave standing between ionospheres, $\omega \sim \Omega_{T N}\left(x^{1}\right)$, and the drift velocity of the source, $\omega_{d}: m \sim \omega / \omega_{d}$. This explains why observed waves have well-defined $m$ values.

2. In accordance with the observations, the phase velocity of the poloidal Alfvén waves coincides with the direction of the proton drift.
3. Despite its transformation from poloidal to toroidal, at a given point in azimuth the wave remains poloidal during a rather large time span $\tau \sim m / \omega \sim \omega_{d}^{-1}$, which corresponds to a sufficiently large angular distance from the source $\phi=\Omega \tau \sim 1$. Besides, in some sense the wave is always poloidal, because the source continues its azimuthal movement, generating the poloidal wave at new points.

4. If the wave attenuation is taken into account (for example, due to finite conductivity of the ionosphere or wave-particle interaction), then the wave does not have enough time to transform into toroidal, and the maximum wave amplitude will correspond to a mixed polarization, depending on the observation point.

5. Besides, the wave damping leads to a localization across the magnetic shells, which corresponds to observational data for the high- $m$ waves. If the azimuthal coordinate is fixed, in the course of time the localization region will shift toward the Earth.

As was mentioned in the Introduction, some of these features of the high- $m$ waves cannot be explained by the theory of drift-bounce instability. It must be noted, however, that the wave-particle energy exchange can lead to a further amplification or attenuation of the wave. Besides, a ponderomotive force can appear, which governs feedback of the generated field upon the generating particles, which can result in the formation of the stationary ring current. However, these questions are far beyond the topic of the present paper.

The most intriguing feature of the wave evolution is the double change of the wave polarization, resulting from the supposed growth of the drift velocity with the radial coordinate; in this case the source is stretched into strips at an acute angle to the magnetic shells. This feature can be used for the verification of the generation of the wave by a moving source as it represents a trademark signature of this mechanism. Certainly, the opposite case, when the drift velocity decreases with the L-shell, seems to be more common. But in this case the evolution is not so interesting, because the angle of the source to the magnetic shells is obtuse now, and the wave polarization is toroidal from the very beginning and remains toroidal till the wave disappears, as in the simplest case of the impulse-generated waves (see, e.g. Klimushkin and Mager, 2004). Thus, in this case we do not have a simple key to distinguish our mechanism from the drift-bounce instability. 


\section{Appendix A}

\section{The derivation of Eq. (7)}

Fourier-transformation of Eq. (7):

$$
\begin{aligned}
& \frac{1}{(2 \pi)^{2}} \int_{-\infty}^{+\infty} d \varphi \int_{-\infty}^{+\infty} d t e^{i \omega t-i m \varphi} \mathcal{L}_{A} \Phi= \\
= & -\frac{1}{(2 \pi)^{2}} \int_{-\infty}^{+\infty} d \varphi \int_{-\infty}^{+\infty} d t e^{i \omega t-i m \varphi} \frac{4 \pi}{c^{2}} \sqrt{g} \frac{\partial}{\partial x^{2}} \frac{\partial}{\partial t} j_{\mathrm{ext}}^{2} .
\end{aligned}
$$

We replace $x^{2}$ by $\varphi$ in $\mathcal{L}_{A}$ and the right side of this equation. Since $g_{1,2}$ and $g$ are independent of $\varphi$ and $t$ then for the left side of this equation we have

$$
\begin{aligned}
& -\frac{\partial}{\partial x^{1}} \frac{\sqrt{g}}{g_{1}} \frac{1}{A^{2}} \frac{\partial}{\partial x^{1}} \frac{1}{(2 \pi)^{2}} \int_{-\infty}^{+\infty} d \varphi \int_{-\infty}^{+\infty} d t e^{i \omega t-i m \varphi} \frac{\partial^{2}}{\partial t^{2}} \Phi \\
& +\frac{\partial}{\partial x^{1}} \frac{\partial}{\partial x^{3}} \frac{g_{2}}{\sqrt{g}} \frac{\partial}{\partial x^{3}} \frac{\partial}{\partial x^{1}} \frac{1}{(2 \pi)^{2}} \int_{-\infty}^{+\infty} d \varphi \int_{-\infty}^{+\infty} d t e^{i \omega t-i m \varphi} \Phi \\
& -\frac{\sqrt{g}}{g_{2}} \frac{1}{A^{2}} \frac{1}{(2 \pi)^{2}} \int_{-\infty}^{+\infty} d \varphi \int_{-\infty}^{+\infty} d t e^{i \omega t-i m \varphi} \frac{\partial^{2}}{\partial t^{2}} \frac{\partial^{2}}{\partial \varphi^{2}} \Phi \\
& +\frac{\partial}{\partial x^{3}} \frac{g_{1}}{\sqrt{g}} \frac{\partial}{\partial x^{3}} \frac{1}{(2 \pi)^{2}} \int_{-\infty}^{+\infty} d \varphi \int_{-\infty}^{+\infty} d t e^{i \omega t-i m \varphi} \frac{\partial^{2}}{\partial \varphi^{2}} \Phi .
\end{aligned}
$$

After integrating by parts we obtain:

$$
\begin{gathered}
\frac{\partial}{\partial x^{1}} \frac{\sqrt{g}}{g_{1}} \frac{\omega^{2}}{A^{2}} \frac{\partial}{\partial x^{1}} \frac{1}{(2 \pi)^{2}} \int_{-\infty}^{+\infty} d \varphi \int_{-\infty}^{+\infty} d t e^{i \omega t-i m \varphi} \Phi \\
+\frac{\partial}{\partial x^{1}} \frac{\partial}{\partial x^{3}} \frac{g_{2}}{\sqrt{g}} \frac{\partial}{\partial x^{3}} \frac{\partial}{\partial x^{1}} \frac{1}{(2 \pi)^{2}} \int_{-\infty}^{+\infty} d \varphi \int_{-\infty}^{+\infty} d t e^{i \omega t-i m \varphi} \Phi \\
-m^{2} \frac{\sqrt{g}}{g_{2}} \frac{\omega^{2}}{A^{2}} \frac{1}{(2 \pi)^{2}} \int_{-\infty}^{+\infty} d \varphi \int_{-\infty}^{+\infty} d t e^{i \omega t-i m \varphi} \Phi \\
-m^{2} \frac{\partial}{\partial x^{3}} \frac{g_{1}}{\sqrt{g}} \frac{\partial}{\partial x^{3}} \frac{1}{(2 \pi)^{2}} \int_{-\infty}^{+\infty} d \varphi \int_{-\infty}^{+\infty} d t e^{i \omega t-i m \varphi} \Phi .
\end{gathered}
$$

Here $1 /(2 \pi)^{2} \int_{-\infty}^{+\infty} d \varphi \int_{-\infty}^{+\infty} d t e^{i \omega t-i m \varphi} \Phi \equiv \Phi_{m \omega}$.

For the right side, after substituting $j_{\mathrm{ext}}^{2}$ in the explicit form (9) we have

$$
-\frac{4 \pi}{c^{2}} \sqrt{g} e n_{0} \omega_{d} \frac{1}{(2 \pi)^{2}} \int_{-\infty}^{+\infty} d \varphi \int_{-\infty}^{+\infty} d t e^{i \omega t-i m \varphi}
$$

$$
\begin{aligned}
& \times \frac{\partial}{\partial \varphi} \frac{\partial}{\partial t} \delta\left(\varphi-\omega_{d} t\right) \Theta(t) \\
& =-m \omega \frac{4 \pi}{c^{2}} \sqrt{g} e n_{0} \omega_{d} \frac{1}{(2 \pi)^{2}} \int_{-\infty}^{+\infty} d t \Theta(t) e^{i \omega t-i m \omega_{d} t} \equiv \tilde{q}_{m \omega} .
\end{aligned}
$$

\section{Appendix B}

\section{The derivation of Eq. (19)}

We solve Eq. (17) by means of the Fourier transform. Let us put

$$
R_{N}\left(x^{1}\right)=\int_{-\infty}^{+\infty} e^{i k x^{1}} R_{N}(k) d k
$$

then

$$
\begin{aligned}
& R_{N}(k)=i m \int_{-\infty}^{k} d k^{\prime} \frac{i q\left(k^{\prime}\right)}{\sqrt{\left(k^{\prime 2}+k_{y}^{2}\right)\left(k^{\prime 2}+k_{y}^{2}\right)}} \\
& \times \exp \left[i\left(k-k^{\prime}\right) x_{T N}^{1}+i k_{y} \Delta_{N}\left(\arctan \frac{k}{k_{y}}-\arctan \frac{k^{\prime}}{k_{y}}\right)\right],
\end{aligned}
$$

where $k_{y}=m / L$,

$q\left(k^{\prime}\right)=\frac{1}{2 \pi} \int_{-\infty}^{+\infty} e^{-i k x^{1}} q\left(x^{1}\right) d x^{1}=$

$$
\begin{aligned}
& =\frac{q_{0}}{(2 \pi)^{2}} \int_{-\infty}^{+\infty} d t^{\prime} e^{i \omega t^{\prime}} \Theta\left(t^{\prime}\right) \int_{-\infty}^{+\infty} d x^{1} e^{-i k^{\prime} x^{1}-i m \Omega_{d} x t^{\prime}}= \\
& =\frac{q_{0}}{2 \pi} \int_{-\infty}^{+\infty} d t^{\prime} e^{i \omega t^{\prime}} \Theta\left(t^{\prime}\right) \delta\left(k^{\prime}+m \Omega_{d} t^{\prime}\right) .
\end{aligned}
$$

Then we integrate over $k^{\prime}$, substitute the expression obtained into Eq. (B1) and introduce new variables $x^{1}=l \xi, k=\kappa / l$. As a result we obtain Eq. (19).

\section{Appendix C}

The derivation of Eq. (23)

After substitution of Eq. (19) into Eq. (22) we obtain the expression for $\mathcal{R}_{N}$ as the integral

$\mathcal{R}_{N}=i q_{0} L \int_{-\infty}^{+\infty} d \kappa \int_{-\infty}^{+\infty} d m \int_{-\infty}^{+\infty} d t^{\prime} \int_{-\infty}^{+\infty} d \omega$ 


$$
\begin{aligned}
& \times \frac{\Theta\left(\kappa+m \Omega t^{\prime}\right) \Theta\left(t^{\prime}\right)}{\sqrt{\left(\kappa^{2}+m^{2} \frac{l^{2}}{L^{2}}\right)\left(\frac{L^{2}}{l^{2}} \Omega^{2} t^{\prime 2}+1\right)}} \\
& \times \exp \left[i \omega\left(t^{\prime}+\frac{m \Omega}{\Omega_{0}} t^{\prime}+\frac{\kappa}{\Omega_{0}}\right)-i m \Omega t^{\prime}+i \kappa(\xi-1)\right. \\
& \left.+i m \delta_{N} \frac{l}{L}\left(\arctan \frac{\kappa L}{m l}+\arctan \Omega t^{\prime} \frac{L}{l}\right)\right] .
\end{aligned}
$$

First we integrate over $\omega$, which gives us $\delta\left(t^{\prime}+\frac{m \Omega}{\Omega_{0}} t^{\prime}+\frac{\kappa}{\Omega_{0}}\right)$ in the subintegral function, then we integrate the expression obtained over $t^{\prime}$. As a result, Eq. (C1) reduces to the simpler form (23) with only two integrations.

\section{Appendix D}

\section{The stationary phase method}

Let us introduce the large parameters $\mu=\Omega_{0} / \Omega \gg 1$ (Since the drift angular velocity is assumed to be much less than the toroidal and poloidal eigenfrequencies), and small parameter $\delta_{N}=\Delta_{N} / l \ll 1$ (since the distance between toroidal and poloidal surfaces $\Delta_{N}$ is much less that the scale of the magnetosphere). Let us find the point of the stationary phase $\left(m_{0}, \kappa_{0}\right)$ through the conditions

$$
\left.\frac{\partial \Psi}{\partial \kappa}\right|_{m_{0}, \kappa_{0}}=0,\left.\quad \frac{\partial \Psi}{\partial m}\right|_{m_{0}, \kappa_{0}}=0 .
$$

After we neglect the terms proportional to the small parameter $\delta_{N}$, we obtain two equations, which determine the position of this point:

$\xi-1+\frac{m_{0}}{m_{0}+\mu}=0$,

$\varphi-\mu \frac{\Omega_{0} t-\kappa_{0}}{\left(m_{0}+\mu\right)^{2}}=0$.

We find from these equations that

$m_{0}=\mu \frac{1-\xi}{\xi}$,

$\kappa_{0}=\mu\left(\Omega t-\varphi \xi^{-2}\right)$

Thus, we find the wave phase: Eq. (26). Further, following the stationary phase method, we find the expression determining the wave amplitude:

$$
\begin{aligned}
& \mathcal{A}_{0}=\widetilde{\mathcal{A}}_{0} \int_{-\infty}^{+\infty} d \kappa \int_{-\infty}^{+\infty} d m \exp \left[i \frac{1}{2} A\left(\kappa-\kappa_{0}\right)^{2}\right. \\
& \left.+i \frac{1}{2} B\left(m-m_{0}\right)^{2}+i C\left(\kappa-\kappa_{0}\right)\left(m-m_{0}\right)\right],
\end{aligned}
$$

where

$$
\widetilde{\mathcal{A}}_{0}=\frac{i q_{0} L \mu \Theta\left(\kappa_{0}+m_{0} \Omega t\right) \Theta\left(\Omega_{0} t-\kappa_{0}\right)}{\sqrt{\left(\frac{L^{2}}{l^{2}}\left(\Omega_{0} t-\kappa_{0}\right)^{2}+\left(\mu+m_{0}\right)^{2}\right)\left(\kappa_{0}+m_{0} \frac{l^{2}}{L^{2}}\right)}},
$$

$$
\begin{aligned}
& A=\left.\frac{\partial^{2} \Psi}{\partial \kappa^{2}}\right|_{m_{0}, \kappa_{0}} \sim \frac{\delta_{N}}{\mu}, \\
& B=\left.\frac{\partial^{2} \Psi}{\partial m^{2}}\right|_{m_{0}, \kappa_{0}} \approx 2 \frac{\xi}{\mu}, \\
& C=\left.\frac{\partial^{2} \Psi}{\partial \kappa \partial m}\right|_{m_{0}, \kappa_{0}} \approx \frac{\xi^{2}}{\mu} \varphi .
\end{aligned}
$$

It is seen that $A \ll C, B$. Thus, we have

$$
\begin{aligned}
& \mathcal{A}_{0}=\widetilde{\mathcal{A}}_{0} \int_{-\infty}^{+\infty} d m e^{i \frac{\xi}{\mu}\left(m-m_{0}\right)^{2}} \int_{-\infty}^{+\infty} d \kappa e^{i \frac{\xi^{2}}{\mu} \varphi\left(\kappa-\kappa_{0}\right)\left(m-m_{0}\right)}= \\
& =\widetilde{\mathcal{A}}_{0} \int_{-\infty}^{+\infty} d m e^{i \frac{\xi}{\mu}\left(m-m_{0}\right)^{2}} 2 \pi \frac{\mu}{\xi^{2}} \delta\left(m-m_{0}\right)=\widetilde{\mathcal{A}}_{0} 2 \pi \frac{\mu}{\xi^{2}} .
\end{aligned}
$$

As a result, after some algebra, we obtain the expression (27) for the wave amplitude.

Acknowledgements. The work is supported by INTAS grant 051000008-7978, RFBR grant 07-05-00185, Program of presidium of Russian Academy of Sciences \#16, and OFN RAS \#16. The work of P. N. Mager was also supported by Russian Science Support Foundation.

Topical Editor I. A. Daglis thanks S. Buchert and L. Ozeke for their help in evaluating this paper.

\section{References}

Akhiezer, A. I., Akhiezer, I. A., Polovin, R. V., Sitenko, A. G., and Stepanov, K. N.: Collective oscillations in a plasma, M.I.T. Press, Cambridge, Massachusetts, 1967.

Akhiezer, A. I., Akhiezer, I. A., Polovin, R. V., Sitenko, A. G., and Stepanov, K. N.: Plasma electrodynamics. Vol. 1: Linear theory (International Series of Monographs in Natural Philosophy, Vol. 68), Oxford, Pergamon Press, 1975.

Allan, W., Poulter, E. M., and Nielsen, E.: STARE observations of a Pc5 pulsations with large azimuthal wave number, J. Geophys. Res., 87, 6163-6172, 1982.

Allan, W., Poulter, E. M., and Nielsen, E.: Pc5 pulsations associated with ring current proton drift: STARE radar observations, Planet Space Sci., 31, 1279-1289, 1983.

Anderson, B. J.: Statistical studies of Pc3-5 pulsations and their relevance for possible source mechanisms of ULF waves, Ann. Geophys., 11, 128-143, 1993, http://www.ann-geophys.net/11/128/1993/.

Baddeley, L. J., Yeoman, T. K., Wright, D. M., Davies, J. A., Trattner, K. J., and Roeder, J. L.: Morning sector drift-bounce resonance driven ULF waves observed in artificially-induced HF radar backscatter, Ann. Geophys., 20, 1487-1498, 2002, http://www.ann-geophys.net/20/1487/2002/.

Baddeley, L. J., Yeoman, T. K., Wright, D. M., Trattner, K. J., and Kellet, B. J.: A statistical study of unstable particle populations in the global ring current and their relation to the generation of high ULF waves, Ann. Geophys., 22, 4229-4241, 2004, http://www.ann-geophys.net/22/4229/2004/. 
Baddeley, L. J., Yeoman, T. K., Wright, D. M., Trattner, K. J., and Kellet, B. J.: On the coupling between unstable magnetospheric particle populations and resonant high $m$ ULF wave signatures in the ionosphere, Ann. Geophys., 23, 567-577, 2005a, http://www.ann-geophys.net/23/567/2005/.

Baddeley, L. J., Yeoman, T. K., and Wright, D. M.: HF doppler sounder measurements of the ionospheric signatures of small scale ULF waves, Ann. Geophys., 23, 1807-1820, 2005b, http://www.ann-geophys.net/23/1807/2005/.

Chisham, G., Orr, D., and Yeoman, T. K.: Observations of a giant pulsation across an extended array of ground magnetometers and on auroral radar, Planet. Space Sci., 40, 953-964, 1992.

Fenrich, F. R., Samson, J. C., Sofko, G., and Greenwald, R. A.: ULF high- and low- $m$ field line resonances observed with the Super Dual Auroral Radar Network, J. Geophys. Res., 100, 21 535$21548,1995$.

Eriksson, P. T. I., Blomberg, L. G., Walker, A. D. M., Glassmeier, K.-H.: Poloidal ULF oscillations in the dayside magnetosphere: a Cluster study, Ann. Geophys., 23, 2679-2686, 2005, http://www.ann-geophys.net/23/2679/2005/.

Eriksson, P. T. I., Blomberg, L. G., and Glassmeier, K.-H.: Cluster satellite observations of $\mathrm{mHz}$ pulsations in the dayside magnetosphere, Adv. Space Res., 38, 1730-1737, 2006.

Glassmeier, K.-H.: Magnetometer array observations of a giant pulsation in the magnetosphere, J. Geophys., 48, 138-147, 1980.

Glassmeier, K.-H.: ULF pulsations, Handbook of Atmospheric Electrodinamics, II, 463-502, 1995.

Glassmeier, K.-H., Buchert, S., Motschmann, U., Korth, A., and Pedersen, A.: Concerning the generation of geomagnetic giant pulsations by drift-bounce resonance ring current instabilities, Ann. Geophys., 17, 338-350, 1999, http://www.ann-geophys.net/17/338/1999/.

Guglielmi, A. V. and Zolotukhina, N. A.: Excitation of Alfvén oscillations of the magnetosphere by the asymmetric ring current, Issled. geomagn. aeron. i fiz. Solntsa, 50, 129-137, 1980 (in Russian).

Gurnett, D. A.: On a remarkable similarity between the propagation of whistlers and the bow wave of a ship, Geophys. Res. Lett., 22, 1865-1868, 1995.

Hughes, W. J., Southwood, D. J., Mauk, B., McPherron, R. L., and Barfield, J. N.: Alfvén waves generated by an inverted plasma energy distribution, Nature, 275, 43-44, 1978.

Karpman, V. I., Meerson, B. I., Mikhailovsky, A. B., and Pokhotelov, O. A.: The effects of bounce resonances on wave growth rates in the magnetosphere, Planet. Space. Sci., 25, 573585, 1977.

Klimushkin, D. Yu.: The propagation of high- $m$ Alfvén waves in the Earth's magnetosphere and their interaction with high-energy particles, J. Geophys. Res., 105, 23 303-23 310, 2000.
Klimushkin, D. Yu.: How energetic particles construct and destroy poloidal high- $m$ Alfvén waves in the magnetosphere, Planet. Space Sci., 55, 722-730, 2007.

Klimushkin, D. Yu. and Mager, P. N.: The spatio-temporal structure of impulse-generated azimuthal small-scale Alfvén waves interacting with high-energy charged particles in the magnetosphere, Ann. Geophys., 22, 1053-1060, 2004, http://www.ann-geophys.net/22/1053/2004/.

Klimushkin, D. Yu., Mager, P. N., and Glassmeier, K.-H.: Toroidal and poloidal Alfvén waves with arbitrary azimuthal wave numbers in a finite pressure plasma in the Earths magnetosphere, Ann. Geophys., 22, 267-288, 2004, http://www.ann-geophys.net/22/267/2004/.

Leonovich, A. S. and Mazur, V. A.: A model equation for monochromatic standing Alfvén waves in the axially-symmetric magnetosphere, J. Geophys. Res., 102, 11 443-11 456, 1997.

Mager, P. N. and Klimushkin, D. Yu.: Spatial localization and azimuthal wave numbers of Alfvén waves generated by driftbounce resonance in the magnetosphere, Ann. Geophys., 23, 3775-3784, 2005, http://www.ann-geophys.net/23/3775/2005/.

Mager, P. N. and Klimushkin, D. Yu.: Generation of Alfvén waves by a plasma inhomogeneity moving in the Earths Magnetosphere, Plasma Physics Reports, 33, 391-398, 2007.

Neubauer, F.: Nonlinear standing Alfvén wave current system at Io - Theory, J. Geophys. Res., 85, 1171-1178, 1980.

Pilipenko, V., Kleimenova, N., Kozyreva, O., Engebretson, M., and Rasmussen, O.: Long-period magnetic activity during the May 15, 1997 storm, J. Atmos. Sol.-Terr. Phys., 63, 489-501, 2001.

Schäfer, S, Glassmeier, K.-H., Eriksson, P. T. I., Pierrard, V., Fornasson, K.-H., and Blomberg, L. G.: Spatial and temporal characteristics of poloidal waves in the terrestrial plasmasphere: A CLUSTER Case Study, Ann. Geophys., 25, 1011-1024, 2007, http://www.ann-geophys.net/25/1011/2007/.

Southwood, D. J.: Low frequency pulsation generation by energetic particles, J. Geomagn. Geoelectr., Suppl. II, 32, 75-88, 1980.

Wright, D. M., Yeoman, T. K., Rae, I. J., Storey, J., Stockton-Chalk, A. B., Roeder, J. L., and Trattner, K. J.: Ground-based and Polar spacecraft observations of a giant $(\mathrm{Pg})$ pulsation and its associated source mechanism, J. Geophys. Res., 106, 10 837-10 852, 2001.

Yeoman, T. K., Wright, D. M., Chapman, P. J., and Stockton-Chalk, A. B.: High-latitude observations of ULF waves with large azimuthal wavenumbers, J. Geophys. Res., 105, 5453-5462, 2000.

Zolotukhina, N. A.: On excitation of Alfvén waves in the magnetosphere by a moving source, Issled. geomagn. aeron. i fiz. Solntsa, 34, 20-23, 1974 (in Russian). 